\title{
Merkel cell carcinoma with kidney metastasis in a 81-year-old man. $A$ rare case report
}

\author{
Aikaterini Anastasiou ${ }^{1}$, Napoleon Moulavasilis ${ }^{1}$, Ioannis Leotsakos ${ }^{1}$, Christos E. Nerantzis ${ }^{2}$, \\ Ioannis Anastasiou ${ }^{1}$ \\ ${ }^{1} 1^{\text {st }}$ University Urology Clinic, Laiko Hospital, Athens Greece; \\ ${ }^{2}$ Forensic Medical Service of Athens, Athens, Greece.
}

\begin{abstract}
Summary Merkel cell carcinoma (MCC) is a primary neuroendocrine carcinoma of the skin. The prognosis of the disease is considered poor. Secondary metastasis is common, however a secondary metastasis to kidney from Merkel cell is a very rare phenomenon. We report a case of a man with a MCC metastasis to the right kidney.

The suggested management is surgery and afterwards a platinum-based chemotherapy.
\end{abstract}

KEY WORDS: Merkel cell carcinoma; Metastasis; Kidney.

Submitted 13 March 2019; Accepted 21 March 2019

\section{INTRODUCTION}

Merkel cell carcinoma (MCC) is a rare non melanoma cutaneous malignancy with a high recurrence and mortality rates $(1,2)$.

It commonly metastasizes to lymph nodes, liver, lung and bone. It occurs mainly in elderly, and people with immunosuppression due to organ transplantation and HIV infection $(1,2)$. Secondary kidney tumors in patients without evidence of disseminated non-renal malignancy are rarely observed (3). We present a rare case of a 81-year-old man with a MCC metastasis to the right kidney.

\section{Case presentation}

A 81-year-old man was presented to our Urology Department ( $1^{\text {st }}$ University Urology Clinic, Laiko hospital, Athens, Greece) after an MRI scan revealed a solid lesion to his right kidney. A biopsy was performed and the histology revealed Merkel Cell Carcinoma (MCC) (Figure 1). The patient, has been diagnosed from MCC in the left submaxillary gland, which in the previous year metastasized in the cervical and parotid glands.

These tumors where managed with surgery, chemotherapy and radiotherapy. MCC is known for its aggressiveness and in our case the kidney was the rare secondary metastasis of the tumor.

The oncology board decided that the patient had to be submitted to a platinum based chemotherapy. Today, 3 years after the last cycle of the chemotherapy our patient is without recurrence.

\section{Discussion}

Merkel cell carcinoma is a primary neuroendocrine carcinoma of the skin (1). It is a highly aggressive tumor with common metastasis to lymph nodes, liver, lung and bone (1). The clinical presentation is a rapidly expanding, $1-2 \mathrm{~cm}$ sized, asymptomatic, red/pink nodule seen on sun-exposed skin (1). Thus it usually affects the head, neck and limbs (1). The mean latency from the primary tumor diagnosis to a systemic metastasis is 2.1 years. Merkel cell carcinoma can be metastasized to every organ, with liver and lungs being the most commonly affected solid organs. Distant dissemination occurs in up to $40-50 \%$ of patients that develop visceral metastasis (4). Our patient first was presented with a submaxillary gland tumor.

The evolution of the MCC in our patient was also rapid and after 3 months he was diagnosed with a cervical gland tumor and lastly after 4 months with a tumor in parotid. One year later an MRI showed a metastasis to the right kidney (Figure 1).

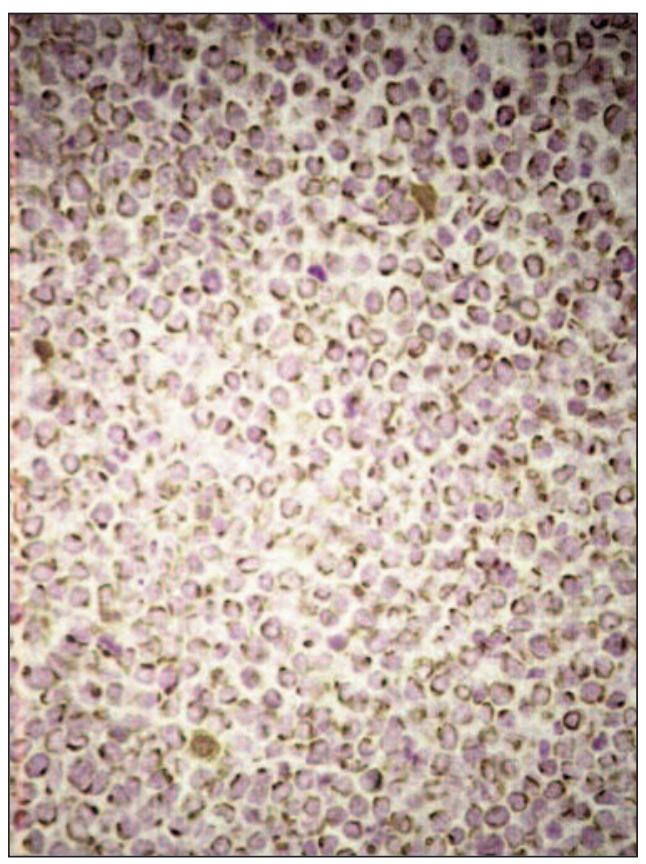

Figure 1. Histology of Merkel cell carcinoma.

No conflict of interest declared. 
Male sex, advanced age, white skin population, vitamin D deficiency, exposure to ultraviolet B and sun are reported to worsen the prognosis. MCC prognosis is also affected by immunosuppression from organ transplant and HIV infection (2).

Diagnosis is based on histological and immunohistochemical analysis.. Histological features include a triad of vesicular nuclei with tiny nucleoli, numerous mitoses and apoptosis, with accompanying lymphovascular invasion (1).

The choice of treatment depends on the characteristics of the disease, the stage at the presentation, the regional lymph node involvement, comorbidities and the performance status of the patient. Surgery is the primary option for patients with locoregional primary MCC.

A combination therapy with adequate surgical removal and selective adjuvant radiotherapy, can reduce the local recurrence rate in a higher percentage (2). However, it is still common for patients with clinically positive lymph nodes to present a high percentage of recurrence in the form of distant disease. Finally, MCC is considered to be a chemotherapy- sensitive tumor especially for the treatment of metastatic MCC (stage IV), and it is mostly indicated to palliate symptoms (2).

Accordingly our patient for the first 10 months, the tumors of the glands where managed with surgery, chemotherapy (Etoposide $100 \times 6$, and Filgastin) and then radiotherapy. When the MRI showed a suspected solid lesion to the right kidney we performed a biopsy and two tumors were found. After the surgery the patient was submitted to a chemotherapy (Platinum $\times 8$ ) and 3 years after the operation is without an evidence of a recurrence

\section{Conclusions}

MCC is an aggressive neoplasm, which usually affects the head and the neck. The disease has an average age of presentation of 72 years. Metastasis are observed in almost half of the patients. The most common are the lungs, liver and bones. A kidney metastasis is very rare in MCC patients, however it is possible to occur.

\section{References}

1. Medhi S, Purandare NC, Dua SG, Gujral S. Bilateral renal metastases in a case of Merkel cell carcinoma. J Cancer Res Ther. 2010; 6:353-5.

2. Schadendorf D, Lebbé C, Zur Hausen A, et al. Merkel cell carcinoma: epidemiology, prognosis, therapy and unmet medical needs. Eur J Cancer. 2017; 71:53-69.

3. Pollheimer VS, Bodo K, Pollheimer MJ, et al. Merkel cell carcinoma metastasizing to the kidney mimicking primary neuroendocrine renal cancer. APMIS. 2007; 115:774-7.

4. Kouzmina M, Koljonen V, Leikola J, et al. Frequency and locations of systemic metastases in Merkel cell carcinoma by imaging. Acta Radiol Open. 2017; 6:1-7.

\section{Correspondence}

Aikaterini Anastasiou, MD

aikatianast@gmail.com

Napoleon Moulavasilis, MD

Napomoul@hotmail.com

Ioannis Leotsakos, $\mathrm{MD}$

j_leot@yahoo.gr

Iohannis Anastasiou, MD

ekati2@otenet.gr

1st University Urology Clinic, Laiko Hospital

Ag.Thoma 17, Athens 11527 (Greece)

Christos E. Nerantzis, MD

theano9@otenet.gr

Forensic Medical Service of Athens, Athens (Greece) 